\title{
Giant tubular adenoma of the accessory breast in the anterior chest wall occurred in a pregnant woman
}

\author{
Yaoyu Huang ${ }^{1 \dagger}$, Hao Zhang ${ }^{1 \dagger}$, Qian Zhou ${ }^{2}$, Lijun Ling $^{1 *}$ and Shui Wang ${ }^{1 *}$
}

\begin{abstract}
Background: Tubular adenoma of the breast is a rare benign epithelial tumor and only a few literatures have been reported; so far, no cases of tubular adenoma occurred in the accessory breast have been reported in the English literature. Clinical presentation and management of our patient are discussed along with a review of the literature on accessory mammary and tubular adenoma.
\end{abstract}

Case presentation: We present a case of 26-year-old woman (gravid 4, para 1) at 37 weeks of pregnancy with rapid enlargement in left anterior chest wall during pregnancy. Physical examination showed the left accessory breast was obviously bigger than the right one that only had a light areola around a small nipple. An elastic, mobile well-circumscribed mass measuring approximately $15 \mathrm{~cm} \times 15 \mathrm{~cm}$ was palpated. Moreover, it was edematous and congestive with an increase in local temperature. The breast ultrasound further demonstrated the mass was a relatively homogeneous solid with short stripe blood flow signal. A single live fetus of 37 weeks gestation was observed by abdominal ultrasound scan. After a $2850 \mathrm{~g}$ male neonate was delivered, the right accessory breast and the mass in left accessory breast were removed. The resected specimen appeared as a solid white elastic mass with a smooth surface and the cut surface was red-grayish. Microscopically, the lesion consisted of tightly packed homogenous glandular structures which are supported by a single layer of myoepithelial cells with sparse intervening stroma.

Conclusions: We describe a very rare case of giant tubular adenoma arising within an accessory breast in the anterior chest wall in a late pregnancy woman. The high concentrations of estrogen, progesterone and prolactin might account for the significant tumor enlargement during pregnancy. To our knowledge, this is the first case of giant tubular adenoma occurred within the accessory breast in the anterior chest wall.

Virtual slides: The virtual slide(s) for this article can be found here: http://www.diagnosticpathology.diagnomx.eu/vs/ 6210811191552106.

Keywords: Tubular adenoma, Accessory breast, Pregnant woman

\section{Background}

Tubular adenoma of the breast is a rare benign epithelial tumor and only a few literatures have been reported, most of them are identified in young women of reproductive age [1], but no cases of tubular adenoma occurred in the accessory breast have been reported in the English literature. Tubular adenoma, also known as pure adenoma, accounts for $0.13-1.7 \%$ of benign breast

\footnotetext{
* Correspondence: pangpangzheng@126.com; ws0801@hotmail.com ${ }^{\dagger}$ Equal contributors

'Department of Breast Surgery, The First Affiliated Hospital with Nanjing Medical University, Nanjing, Jiangsu 210029, China

Full list of author information is available at the end of the article
}

lesions [2]. It was first recognized as a distinctive entity in 1968 by Persaud who referred to it as "fibroadenoma with predominantly glandular component" [3]. Clinically, it is difficult to differentiate tubular adenoma from other benign lesions (fibroadenoma) and from malignant breast cancer (tubular carcinoma) radiographically [4], and surgical excision is often necessary in order to reach a precise diagnosis and a definitive treatment [5]. We herein describe a very rare case of a rapidly enlarging mass arising within an accessory mammary gland in the anterior chest wall in a 26-year-old woman at 37 weeks of pregnancy with successful pregnancy outcome. 


\section{Case presentation}

A 26-year-old woman (gravid 4, para 1) at 37 weeks of pregnancy with rapid enlargement in left anterior chest wall during pregnancy was admitted to our breast surgery unit. She first noticed a peanut size palpable accessory breast lesion at 10 weeks of pregnancy and during pregnancy the mass gradually increased along with pain. A similar symptom occurred in her last pregnancy 5 years ago, but the mass in left anterior chest wall was limited in the peanut size during the whole gestation period. A delayed surgical intervention was suggested. But the lump gradually regressed and disappeared after delivery.

On breast examination, two breasts looked symmetric with a couple accessory breasts below. The left accessory breast was obviously bigger than the right one that only had a light areola around a small nipple. An elastic, mobile well-circumscribed mass measuring approximately $15 \mathrm{~cm} \times 15 \mathrm{~cm}$ was palpated on physical examination of the left accessory breast. Moreover, it was edematous and congestive with an increase in local temperature (Figure 1). The breast ultrasound further demonstrated the mass was a relatively homogeneous solid with short stripe blood flow signal. A single live fetus of 37 weeks gestation was observed by abdominal ultrasound scan. So mammography and dynamic contrast-enhanced Magnetic Resonance Imaging(MRI) were not performed. The patient also refused the core needle biopsy.

Due to progressive enlargement of the lump, diabrosis with bleeding occurred on the third day after admission. Since the fetus had been mature in the 37th week of gestation, we decided to perform the excision of the mass and accessory breasts after caesarean section. After a $2850 \mathrm{~g}$ male neonate was delivered, the right accessory breast and the mass with left accessory breast were

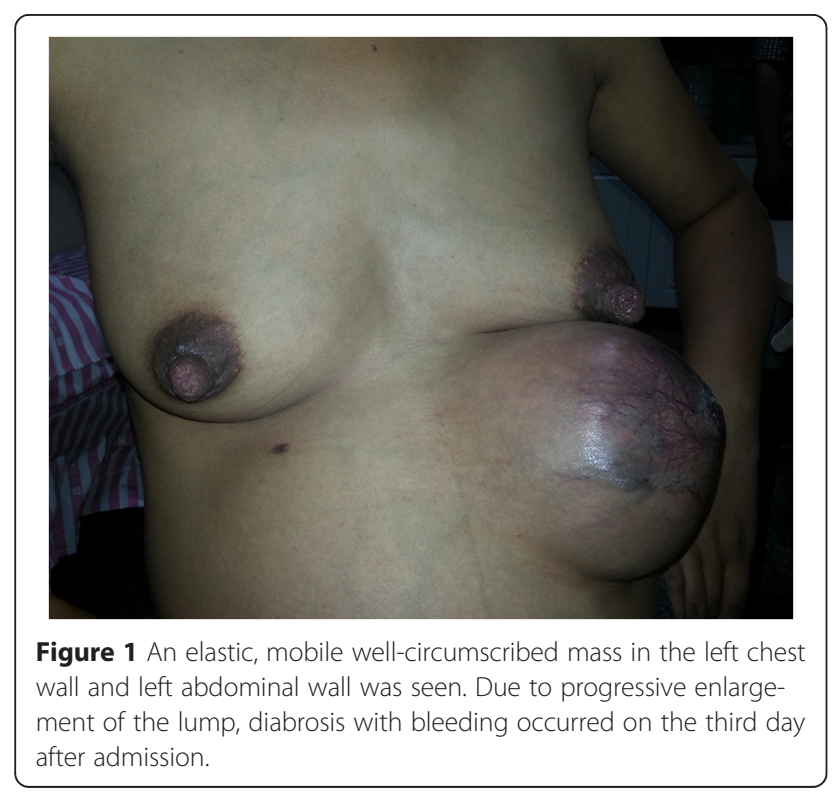

removed. The pathological result came out with a giant tubular adenoma of $15 \mathrm{~cm} \times 15 \mathrm{~cm} \times 12 \mathrm{~cm}$, the resected specimen appeared as a solid white elastic mass with a smooth surface. The cut surface was red-grayish. No hemorrhage or necrosis was present (Figure 2). Microscopically, the lesion consisted of tightly packed homogenous glandular structures which were supported by a single layer of myoepithelial cells with sparse intervening stroma (Figure 3). Milk overflowed from the left chest wall incision three days after operation and cured two days after the administration of bromocriptine. The postoperative course was uneventful and she discharged in good condition in two weeks.

\section{Discussion}

Numerous cases of masses arising in accessory breast have been reported, including both benign and malignant lesions. The most common lump identified in accessory breast tissue is fibroadenoma, and there are scattered case reports of other tumors including phyllodes tumor and mammary carcinoma [6]. Most of them take place in the axilla, in accordance with the incidence of accessory breast tissue. Tubular breast adenoma is a rare benign epithelial lesion which is most commonly found in young women of reproductive age [1]. Some scholars have reported a few cases and described them as elastic-hard, smooth-surfaced, freely movable and well-circumscribed masses without associated skin or nipple alterations [1,3,7-11]. The clinical features and the ultrasound examination are similar to those of fibroadenoma and nonspecific. Therefore, pathological examination is usually necessary in order to reach a precise diagnosis and a definitive treatment. Histologically, tubular adenoma is characterized by the presence of tightly

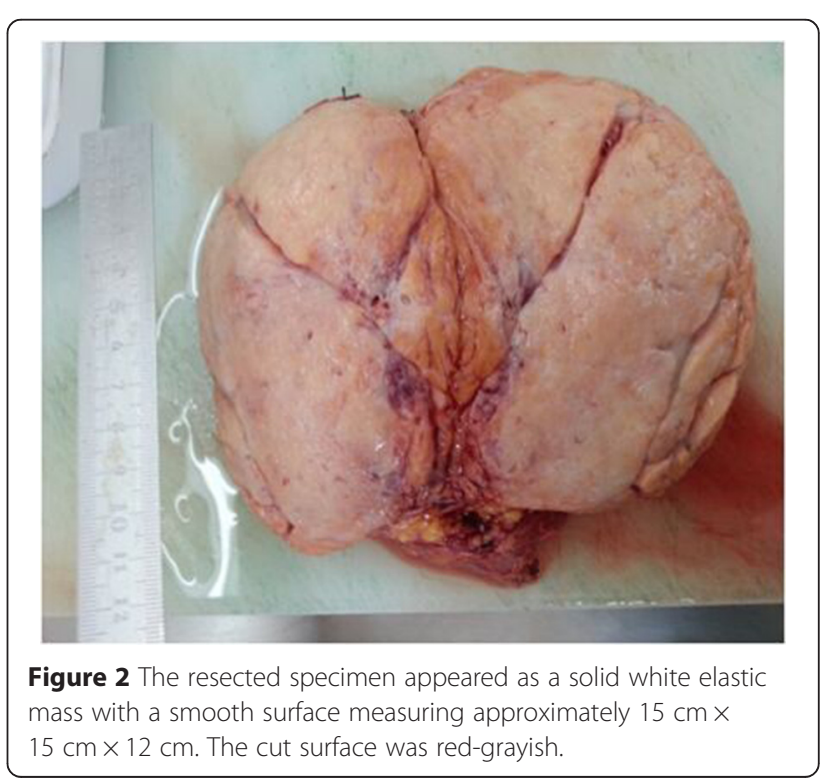



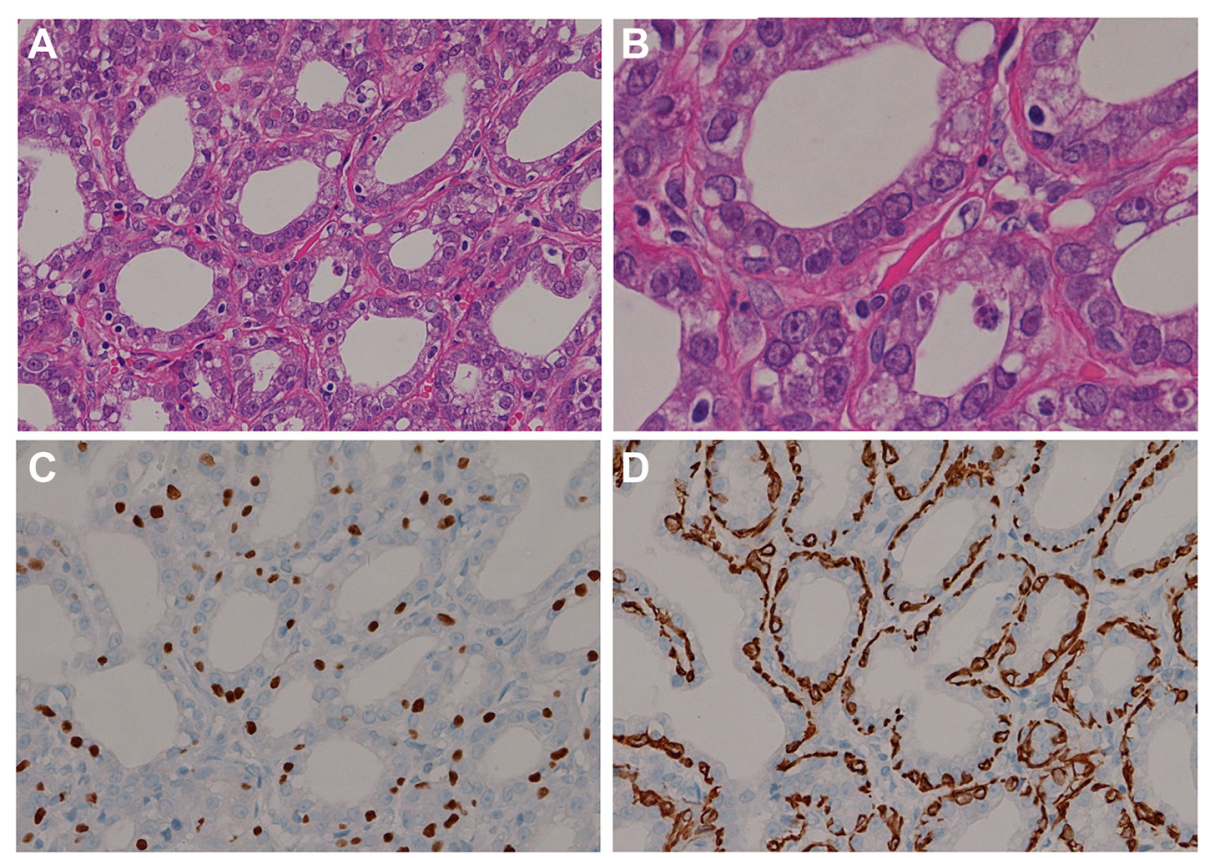

Figure 3 Microscopically, the lesion consisted of tightly packed tubular or acinar structures that were very regular in size and shape with sparse intervening stroma and the glandular structures were supported by a single layer of myoepithelial cells. $(\mathbf{A}$ : H\&E $\times 200$, B: H\&E $\times 200$, C: $p 63 \times 200$, D: calponin $\times 200)$.

packed tubular or acinar structures that are very regular in size and shape with sparse intervening stroma on the contrary to firoadenoma which contains abundant stroma [12,13]. However, sometimes tubular adenoma and fibroadenoma could coexist in one lesion, suggesting that the pathogenesis is closely related [11]. Apart from fibroadenoma, phyllodes tumor which also clinically present as painless freely movable well-defined breast masses should be considered as another differential diagnosis due to the possibility of malignancy [14]. In rare cases, the elastic-hard and rough-surfaced mass could also be the presence of malignancy, especially a mass with rapid enlargement in elderly post-menopausal women [15]. Soo MS compared the imaging features of 17 patients and found that tubular adenoma may resemble a malignancy in patients who were 38 years old or older [4]. Awareness of this may be helpful in preventing aggressive treatment.

Although there is no evidence for the correlation between the occurrence of tubular adenoma and pregnancy, tubular breast adenoma increases in size during pregnancy and could show lactational histologic changes [16]. The high concentrations of estrogen, progesterone and prolactin which promote the growth of ducts and the formation of tubuloalveolar structures might be a reason for the significant tumor enlargement in this period.

During gestation and lactation, the breast undergoes extensive changes and could create diagnostic challenges.
Due to high sensitivity and specificity, ultrasound imaging was concerned to be the first choice [17]. Besides, MRI is considered to be adopted, although some literatures reports that Gadolinium-based contrast medium can pass through the placenta [18]. In the diagnostic process, fineaspiration biopsy (FNAB) and core biopsy remains an increasing important diagnostic tool to evaluate the mass. Except for injuring the pregnancy and the fetus, FNAB and core biopsy provides a certain amount of samples for pathological examination. However, it is noticeable that the false-positive rate and the false-negative rate of the pathological findings is higher due to the breast/accessory breast changes in pregnancy [19], furthermore, core biopsy could induce fistula formation [20]. Breast or accessory breast surgery can be safely performed in whole gestation stage. However, because the risk of spontaneous abortion increases in first trimester [21], we suggest operation should be performed after the first trimester of gestation.

In this case, leakage of milk from the left chest wall incision happened three days after the stimulation of breastfeeding because of the injury to the milk duct during the operation. To avoid this, it is important to be more careful when deal with the edge of tumor nearby normal breast tissues. Some more powerful surgical instruments which could seal the duct also may be helpful. Cessation of breast feeding is necessary along with pharmacological suppression of lactation to prevent continuous milk overflowing from the incision. 


\section{Conclusions}

We describe a very rare case of giant tubular adenoma arising within an accessory breast in the anterior chest wall in a late pregnancy woman. The high concentrations of estrogen, progesterone and prolactin might account for the significant tumor enlargement during pregnancy. To our knowledge, this is the first case of giant tubular adenoma occurred within the accessory breast in the anterior chest wall. Doctors should be aware that diseases of the breast may also arise in accessory mammary tissue no matter how rare the disease is.

\section{Consent}

Written informed consent was obtained from the patient for publication of this case report and any accompanying images. A copy of the written consent is available for review by the Editor-in-Chief of this journal.

\section{Abbreviations}

MRI: Magnetic Resonance Imaging; FNAB: Fine-aspiration biopsy; H\&E: Hematoxylin-eosin staining.

\section{Competing interests}

The authors declare that they have no competing interests.

\section{Authors' contributions}

$\mathrm{YH}$ and $\mathrm{HZ}$ made contributions to acquisition of clinical data, and drafted the manuscript. They are joint first co-authors and made an equal contribution to this work. QZ did the analysis of the histological features by H\&E and immunohistochemistry. SW and LL revised manuscript critically for important intellectual content and had given final approval of the version to be published. All authors read and approved the final manuscript.

\section{Acknowledgements}

The authors would like to thank Tiansong Xia and Wenbin Zhou for the specialist advice on the layout of the paper. Furthermore, The authors are grateful to Zhao Liu for the assistance with the language editing of the paper.

This work was supported partly by the National Natural Science Foundation of China (81071753 and 81172502), the Natural Science Foundation of Jiangsu Province (BK2009438).

\section{Author details}

'Department of Breast Surgery, The First Affiliated Hospital with Nanjing Medical University, Nanjing, Jiangsu 210029, China. ${ }^{2}$ Department of Breast Surgery, Nanjing Maternity and Child Health Care Hospital Affiliated to Nanjing Medical University, Nanjing, Jiangsu, China.

Received: 29 December 2014 Accepted: 28 April 2015

Published online: 04 June 2015

\section{References}

1. Salemis NS, Gemenetzis G, Karagkiouzis G, Seretis C, Sapounas K, Tsantilas V, et al. Tubular adenoma of the breast: a rare presentation and review of the literature. J Clin Med Res. 2012;4(1):64-7.

2. Bocker W. WHO classification of breast tumors and tumors of the female genital organs: pathology and genetics. Verh Dtsch Ges Pathol. 2002:86:116-9.

3. Persaud V, Talerman A, Jordan R. Pure adenoma of the breast. Arch Pathol. 1968:86(5):481-3.

4. Soo MS, Dash N, Bentley R, Lee LH, Nathan G. Tubular adenomas of the breast: imaging findings with histologic correlation. AJR Am J Roentgenol. 2000;174(3):757-61.
5. Rovera F, Ferrari A, Carcano G, Dionigi G, Cinquepalmi L, Boni L, et al. Tubular adenoma of the breast in an 84-year-old woman: report of a case simulating breast cancer. Breast J. 2006;12(3):257-9.

6. Page RN, Dittrich L, King R, Boulos F, Page DL. Syringomatous adenoma of the nipple occurring within a supernumerary breast: a case report. J Cutan Pathol. 2009;36(11):1206-9.

7. Gutermuth J, Audring H, Voit C, Haas N. Primary carcinoma of ectopic axillary breast tissue. J Eur Acad Dermatol Venereol. 2006;20(2):217-21.

8. Val-Bernal JF, Gonzalez-Vela MC, De Grado M, Garijo MF. Sclerotic fibroma (storiform collagenoma)-like stroma in a fibroadenoma of axillary accessory breast tissue. J Cutan Pathol. 2012;39(8):798-802.

9. Gilmore HT, Milroy M, Mello BJ. Supernumerary nipples and accessory breast tissue. S D J Med. 1996:49(5):149-51.

10. Shioi Y, Nakamura S, Kawamura S, Kasami M. Nipple adenoma arising from axillary accessory breast: a case report. Diagn Pathol. 2012;7:162.

11. Hertel BF, Zaloudek C, Kempson RL. Breast adenomas. Cancer. 1976;37(6):2891-905

12. Irshad A, Ackerman SJ, Pope TL, Moses CK, Rumboldt T, Panzegrau B. Rare breast lesions: correlation of imaging and histologic features with $\mathrm{WHO}$ classification. Radiographics. 2008;28(5):1399-414

13. Guray M, Sahin AA. Benign breast diseases: classification, diagnosis, and management. Oncologist. 2006;11(5):435-49.

14. Belkacemi Y, Bousquet G, Marsiglia H, Ray-Coquard I, Magne N, Malard Y et al. Phyllodes tumor of the breast. Int J Radiat Oncol Biol Phys. 2008;70(2):492-500.

15. Nishimori H, Sasaki M, Hirata K, Zembutsu H, Yasoshima T, Fukui R, et al. Tubular adenoma of the breast in a 73-year-old woman. Breast Cancer. 2000;7(2):169-72.

16. Palnaes Hansen C, Fahrenkrug L, Hastrup N. Tubular adenoma of the breast in a pregnant girl: report on a case. Eur J Pediatr Surg. 1991;1(6):364-5.

17. Navrozoglou I, Vrekoussis T, Kontostolis E, Dousias V, Zervoudis S, Stathopoulos EN, et al. Breast cancer during pregnancy: a mini-review. Eur J Surg Oncol. 2008:34(8):837-43.

18. Oto A, Ernst R, Jesse MK, Chaljub G, Saade G. Magnetic resonance imaging of the chest, abdomen, and pelvis in the evaluation of pregnant patients with neoplasms. Am J Perinatol. 2007;24(4):243-50.

19. Amant F, von Minckwitz G, Han SN, Bontenbal M, Ring AE, Giermek J, et al. Prognosis of women with primary breast cancer diagnosed during pregnancy: results from an international collaborative study. J Clin Oncol. 2013;31(20):2532-9.

20. Schackmuth EM, Harlow CL, Norton LW. Milk fistula: a complication after core breast biopsy. AJR Am J Roentgenol. 1993;161(5):961-2.

21. Loibl S, von Minckwitz G, Gwyn K, Ellis P, Blohmer JU, Schlegelberger B, et al. Breast carcinoma during pregnancy. International recommendations from an expert meeting. Cancer. 2006;106(2):237-46.

\section{Submit your next manuscript to BioMed Central and take full advantage of:}

- Convenient online submission

- Thorough peer review

- No space constraints or color figure charges

- Immediate publication on acceptance

- Inclusion in PubMed, CAS, Scopus and Google Scholar

- Research which is freely available for redistribution 